\title{
MicroRNA-143 and -145 modulate the phenotype of synovial fibroblasts in rheumatoid arthritis
}

\author{
Bong-Ki Hong ${ }^{1}$, Sungyong You ${ }^{2}$, Seung-Ah Yoo ${ }^{1}$, Dohyun Park ${ }^{1}$, Daehee Hwang ${ }^{3}$, Chul-Soo Cho ${ }^{1,4}$ \\ and Wan-Uk Kim ${ }^{1,4}$
}

Fibroblast-like synoviocytes (FLSs) constitute a major cell subset of rheumatoid arthritis (RA) synovia. Dysregulation of microRNAs (miRNAs) has been implicated in activation and proliferation of RA-FLSs. However, the functional association of various miRNAs with their targets that are characteristic of the RA-FLS phenotype has not been globally elucidated. In this study, we performed microarray analyses of miRNAs and mRNAs in RA-FLSs and osteoarthritis FLSs (OA-FLSs), simultaneously, to validate how dysregulated miRNAs may be associated with the RA-FLS phenotype. Global miRNA profiling revealed that miR-143 and miR-145 were differentially upregulated in RA-FLSs compared to OA-FLSs. miR-143 and miR-145 were highly expressed in independent RA-FLSs. The miRNA-target prediction and network model of the predicted targets identified insulinlike growth factor binding protein 5 (IGFBP5) and semaphorin 3A (SEMA3A) as potential target genes downregulated by miR-143 and miR-145, respectively. IGFBP5 level was inversely correlated with miR-143 expression, and its deficiency rendered RA-FLSs more sensitive to TNF $\alpha$ stimulation, promoting IL- 6 production and NF-kB activity. Moreover, SEMA3A was a direct target of miR-145, as determined by a luciferase reporter assay, antagonizing VEGF $_{165}$-induced increases in the survival, migration and invasion of RA-FLSs. Taken together, our data suggest that enhanced expression of miR-143 and miR-145 renders RA-FLSs susceptible to TNF $\alpha$ and VEGF $_{165}$ stimuli by downregulating IGFBP5 and SEMA3A, respectively, and that these miRNAs could be therapeutic targets.

Experimental \& Molecular Medicine (2017) 49, e363; doi:10.1038/emm.2017.108; published online 4 August 2017

\section{INTRODUCTION}

Rheumatoid arthritis (RA) is an autoimmune disease that is characterized by chronic inflammatory cell infiltration and pannus formation in synovial tissues that lead to the destruction of articular cartilage and bone. In RA joints, various inflammatory cells become activated via a variety of cytokines and chemokines as well as via cell-cell contact. ${ }^{1}$ Among various cell types, fibroblast-like synoviocytes (FLSs) are the most abundant resident cells in the synovial membrane and play critical roles in the pathogenesis of RA. FLSs in an RA patient (RA-FLSs) have tumor-like features, including an increased proliferation rate, anti-apoptotic ability and pro-migratory and pro-invasive properties that cause pannus formation and joint destruction. ${ }^{2,3}$ Moreover, RA-FLSs produce large amounts of pro-inflammatory cytokines, including interleukin-1 $\beta$ (IL-1 $\beta$ ), IL- 6 and tumor necrosis factor $\alpha(\mathrm{TNF} \alpha)$, that contribute to the perpetuation of chronic inflammation. ${ }^{2}$
MicroRNAs (miRNAs) have emerged as key regulators of a broad spectrum of cellular functions, such as proliferation, differentiation and apoptosis, that are associated with the pathogenesis of various autoimmune diseases. ${ }^{4}$ Evidence is emerging that miRNA expression is dysregulated in RA-FLSs, which may be responsible for various pathologic processes involving RA. ${ }^{5-8}$ For example, miR-126 has been identified as a regulator of phosphatidylinositol 3-kinase (PI3K)/AKT in RA-FLSs, an important signaling molecule mediating cell proliferation and apoptosis. ${ }^{5}$ In addition, miR-20a has been found to have two target genes in FLSs: thioredoxin interacting protein (TXNIP) and apoptosis signal-regulating kinase (ASK1). 6,7 These mediate NLRP3-inflammasome and TLR4dependent cytokine release by FLSs, respectively. ${ }^{6,7}$ miR-221 has also been identified as a regulator for increased migration and invasion of RA-FLSs. ${ }^{8}$ Nevertheless, global and integrated analyses of miRNA and mRNA expression levels, which can

\footnotetext{
${ }^{1}$ Center for Integrative Rheumatoid Transcriptomics and Dynamics, The Catholic University of Korea, Seoul, Korea; ${ }^{2}$ Division of Urology, Department of Surgery and Biomedical Sciences, Cedars-Sinai Medical Center, Los Angeles, CA, USA; ${ }^{3}$ Department of New Biology and Center for Plant Aging Research, Institute for Basic Science, Daegu Gyeongbuk Institute of Science and Technology, Daegu, Republic of Korea and ${ }^{4}$ Division of Rheumatology, Department of Internal Medicine, The Catholic University of Korea, Seoul, Korea

Correspondence: Dr W-U Kim, Division of Rheumatology, Department of Internal Medicine, The Catholic University of Korea, School of Medicine, St Mary's Hospital, 222 Banpo-daero, Seocho-gu, Seoul 06591, Korea.
}

E-mail: wan725@catholic.ac.kr

Received 30 January 2017; accepted 19 February 2017 
address pathologic features of RA-FLSs, have not been systematically performed.

In the present study, we simultaneously profiled the global expression of miRNAs and mRNAs in FLSs through microarray analysis and found previously unidentified miR-143 and miR-145 to be differentially upregulated in RA-FLSs compared to FLSs from osteoarthritis patients (OA-FLSs). We validated that miR-143 and miR-145 were highly expressed in independent RA-FLSs. Moreover, miR-145 expression in RA-FLSs was increased by transforming growth factor $\beta$ (TGF $\beta$ ). By using the miRNA target prediction and network model of the predicted targets, we selected two potential targets, insulinlike growth factor binding protein 5 (IGFBP5) and semaphorin 3A (SEMA3A), that could be downregulated by miR-143 and miR-145, respectively. In in vitro functional tests, IGFBP5 deficiency induced RA-FLS sensitivity to TNF $\alpha$ stimulation and promoted IL- 6 production and NF- $\kappa$ B activity. Moreover, the 3'-UTR of SEMA3A mRNA was shown to be a direct target of miR-145, as determined by a luciferase reporter assay, and recombinant SEMA3A antagonized $\mathrm{VEGF}_{165}$-induced increases in survival, migration and invasion of RA-FLSs. Collectively, our data suggest that enhanced miR-143 and miR-145 expression renders RA-FLSs susceptible to TNF $\alpha$ and VEGF $_{165}$ stimuli by downregulating IGFBP5 and SEMA3A, respectively. Thus, inhibition of miR-143 and miR-145 could lead to a reduction in RA-FLS pathogenic potential.

\section{MATERIALS AND METHODS}

\section{Isolation and culture of FLSs}

FLSs were isolated from the synovial tissues of patients with RA or OA and cultured in Dulbecco's modified Eagle medium (DMEM, 12800-017, Gibco, ThermoFisher Scientific, Grand Island, NY, USA) supplemented with $10 \%$ fetal bovine serum (FBS), $6.0 \mathrm{~g}^{-1}$ of HEPES (0511-1KG, Amresco, Solon, OH, USA), $0.4 \mathrm{~g} \mathrm{l}^{-1}$ of L-glutamine (0374-500G, Amresco), $3.7 \mathrm{gl}^{-1}$ of sodium bicarbonate (0865-1KG, Amresco), $1 \times$ of antibiotics (15240-062, Gibco), $1 \times$ of MEM non-essential amino acids (11140-050, Gibco), and $1 \times$ of 2-mercaptoethanol (24985-023, Gibco). Experiments were performed between passages 3 and 7 of the FLSs.

\section{Microarray analysis for miRNA and mRNA profiling}

We prepared total RNA independently from two replicate samples for miRNA and three replicate samples for mRNA using miRNeasy (217004, Qiagen GmbH, Hilden, Germany) and RNeasy (74104, Qiagen) mini kits, respectively. The RNA integrity was assessed using a Bioanalyzer 2100 (Agilent Technologies, Santa Clara, CA, USA). The RNA integrity number (PRN) was close to 10 for all samples. RNA was then amplified and hybridized with an Agilent-019118 Human miRNA Microarray 2.0 G4470B (for miRNA) and an Illumina HumanHT-12 v4.0 Expression BeadChip (Illumina, San Diego, CA, USA for mRNA), according to the manufacturer's standard protocols. The probe intensities were normalized using the quantile normalization procedure. ${ }^{9}$ The data were deposited into the GEO database (GEO accession ID, miRNA: GSE91026, mRNA: GSE49604).

\section{Integrative statistical testing}

For the four comparisons of RA-FLSs versus OA-FLSs, RA-FLSs+IL1 $\beta$ versus RA-FLSs, OA-FLSs+IL1 $\beta$ versus OA-FLSs, and RA-FLSs+IL1 $\beta$ versus OA-FLSs+IL1 $\beta$, we used the integrated statistical testing method previously reported. ${ }^{10}$ Briefly, for each gene, an adjusted $P$-value was computed by performing a two-tailed $t$-test and $\log 2$ median ratio test using the empirical distributions that were estimated by random permutations of the samples. The two sets of $P$-values from the individual tests were combined to compute the overall $P$-values using Stouffer's method. ${ }^{11}$ Finally, differentially expressed genes (DEGs) were selected that have (1) $P<0.05$ and (2) absolute $\log 2$-fold changes larger than 1.34. To determine the cutoff value for $\log 2$-fold changes, we computed log2-fold changes of randomly permuted samples, fitted a Gaussian distribution to the random $\log 2$-fold changes, and then calculated the 2.5th percentile corresponding to $\alpha=0.05$ (log2-fold-change cutoff $=1.34$ ). The DEGs were grouped into six clusters using hierarchical clustering. The cellular processes represented by the genes in each cluster were identified as the GO biological processes (GOBPs) having $P<0.1$ (the default cutoff value in the DAVID software). ${ }^{12}$

\section{Hierarchical clustering of DEGs}

From the comparisons of RA-FLS versus OA-FLS and RA-FLS+IL1 $\beta$ versus OA-FLS+IL1 $\beta$, we identified a total of 470 DEGs. We then categorized the DEGs into two groups of upregulated and downregulated DEGs. For the DEGs in each group, we further performed hierarchical clustering using Euclidean distance as the dissimilarity measure and the average linkage method for clusters for upregulated and downregulated DEGs in all compared groups.

\section{Prediction of target genes}

We refined lists of predicted miR-143 and miR-145 target genes through comparisons between the DEG list and network analysis. In addition, we referred to the context score from the TargetScan 6.0 database. A Venn diagram was drawn by Bioinformatics \& Evolutionary genomics (http://bioinformatics.psb.ugent.be/webtools/ Venn/),

Reconstruction of miR-143 and miR-145 target gene network To reconstruct a network model for the 1081 genes (404 genes of miR-143 targets, 731 genes of miR-145 and 54 shared genes) that were the predicted targets of miR-143 and/or miR-145, we first collected all protein-protein interactions (PPIs) stored in HPRD (http://www. hprd.org/), BioGRID (https://thebiogrid.org/), STRING (http://stringdb.org/) and KEGG (http://www.genome.jp/kegg/), and then combined all PPIs from the four databases into one list of PPIs. We then used Cytoscape version 3.2.0 software (http://www.cytoscape.org/) to display these PPIs for the 1081 genes as a network model.

\section{Transfection of miRNA inhibitors and short interfering RNAs}

FLSs were seeded at $1 \times 10^{5}$ cells per well in a six-well cell culture plate (Nunc, Thermo Scientific, Rockford, IL, USA). After $24 \mathrm{~h}$, the cells were transfected with $50 \mathrm{~nm}$ of miScript miRNA inhibitors (Negative control: 1027271, hsa-miR-145: MIN0000437, Qiagen) or $10 \mathrm{~nm}$ of short interfering RNAs (siRNA) for IGFBP5 (sc-39591, Santa Cruz Biotechnology, Santa Cruz, CA, USA) with Lipofectamine 2000 (11668-027, Invitrogen, Carlsbad, CA, USA). Transfection efficiency was validated by quantitative real-time polymerase chain reaction (PCR). 
Quantitative real-time PCR analysis

Total RNA samples in FLSs were prepared with TRIzol Reagent (15596018, Ambion, Austin, TX, USA) according to the manufacturer's instruction. Extracted RNA was stored at $-80^{\circ} \mathrm{C}$ before use. For miRNA isolation from synovial fluids, an miRNeasy Serum/ Plasma Kit (217184, Qiagen) was used. To determine the miRNA and mRNA levels, $1 \mu \mathrm{g}$ of RNA was synthesized into cDNA using the miScript II RT Kit (218161, Qiagen). The miRNA levels were assessed by real-time PCR using miScript SYBR Green PCR Kit (218073, Qiagen) with CFX96 (Bio-Rad, Hercules, CA, USA). The mRNA levels were assessed by real-time PCR using iQ SYBR Green Supermix (170-8880, Bio-Rad). The gene-specific primers used are listed in Supplementary Table 1.

\section{MTT assay}

The viability of FLSs was assessed by the MTT (3-[4,5dimethylthiazol-2-yl]-2, 5 diphenyl tetrazolium bromide) assay, as described previously. ${ }^{10}$

\section{Apoptosis assay}

FLS apoptosis was determined using the APOPercentage apoptosis assay kit (Bicolor, Newtownabbey, UK). Digital images of APOPercentage dye-labeled cells, which appear bright pink against the white background under a light microscope, were used to quantify the apoptotic cell numbers.

\section{Invasion and migration assay}

For wound migration assays, FLSs were seeded at $1.5 \times 10^{5}$ cells per well in a six-well plate. After $24 \mathrm{~h}$, the cells were scratched with a pipette tip and then incubated in 1\% FBS DMEM with $200 \mathrm{ng} \mathrm{ml}^{-1}$ of recombinant human VEGF $_{165}$ (293-VE, R\&D Systems, Minneapolis, $\mathrm{MN}$, USA) in the presence or absence of recombinant human SEMA3A (1250-S3, R\&D Systems). After $12 \mathrm{~h}$ of incubation, FLS migration was quantified by counting the cell numbers that had moved beyond a reference line.

An FLS invasion assay was performed in BioCoat Matrigel Invasion Chambers (354480, Thermo Science). FLSs were seeded at $1 \times 10^{5}$ cells per well in a six-well plate with $500 \mu$ of $0.1 \%$ FBS DMEM and $200 \mathrm{ng} \mathrm{ml}^{-1}$ of recombinant human $\mathrm{VEGF}_{165}$ in the presence or absence of recombinant SEMA3A $\left(100 \mathrm{ng} \mathrm{ml}^{-1}\right)$. After $12 \mathrm{~h}$, the non-invading cells were removed by scrubbing with a cotton-tipped swab, and the cells on the lower surface of the membrane were stained with Diff-Quik stain (Baxter Diagnostics, Deerfield, IL, USA). For quantification, the cells were manually counted in eight random fields. After staining, the images were captured by a slide scanner (Pannoramic MIDI, 3DHISTECH Ltd, Budapest, Hungary).

\section{Enzyme-linked immunosorbent assay for IL-6}

Cultured media from FLS were collected and stored at $-80^{\circ} \mathrm{C}$. The IL- 6 concentrations in the culture supernatants were measured by a Human IL-6 Duoset ELISA kit (dy206, R\&D Systems) according to the manufacturer's instructions.

\section{Immunocytochemistry}

For immunocytochemistry staining, RA-FLSs were seeded at $2 \times 10^{3}$ cells per well on eight-well glass chamber slides (154534, Nunc, Thermo Scientific). One hour after TNF $\alpha$ stimulation $\left(0.1 \mathrm{ng} \mathrm{ml}^{-1}\right.$, 210-TA, R\&D Systems), the cells were fixed with chilled fresh methanol and permeabilized with PBS-T containing 0.25\% Triton $\mathrm{X}-100$. The slides were then treated with a blocking buffer (1\% bovine serum albumin in PBS-T) and incubated overnight with anti-p65 antibody (ab7970, Abcam, Cambridge, UK) at $4{ }^{\circ} \mathrm{C}$. After rinsing with PBS-T, the slides were incubated again with goat anti-rabbit IgG secondary antibody and then with Alexa Fluor 488 conjugate (A-11008, Invitrogen) for $30 \mathrm{~min}$ and mounted using mounting medium with DAPI. The mounted slides were examined using a confocal laser scanning microscope (LSM 510 Meta, Zeiss, Oberkochen, Germany).

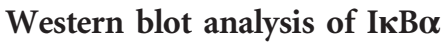

RA-FLSs were seeded at $1 \times 10^{5}$ cells per well in a six-well plate. After siRNA transfection or treatment with recombinant human IGFBP5, the cells were stimulated with $0.1 \mathrm{ng} \mathrm{ml}^{-1}$ of TNF $\alpha$ for the indicated times $(0,1,5,10,15$ and $30 \mathrm{~min})$. The cells were then lysed with $100 \mu \mathrm{l}$ of $1 \times$ sodium dodecyl sulfate sample buffer and incubated at $95^{\circ} \mathrm{C}$ for $5 \mathrm{~min}$. Insoluble material was removed by centrifugation at $12000 \mathrm{~g}$ for $15 \mathrm{~min}$ at $4{ }^{\circ} \mathrm{C}$. Electrophoresis was carried out using sodium dodecyl sulfate-10\% polyacrylamide gels electrophoresis, and the resolved proteins were transferred to nitrocellulose membranes. Membranes were incubated with antibodies to I $\mathrm{\kappa B} \alpha$ (sc-371, Santa Cruz Biotechnology). $\beta$-Actin was used as an internal control. Membranes were then visualized using an enhanced chemiluminescence technique. The resulting films were scanned, and the optical densities were quantified using ImageQuant LAS 4000 (GE Healthcare, Chicago, IL, USA) and ImageJ software.

\section{Reporter vector construction}

The whole SEMA3A 3'-UTR (3 kilobase pairs) was cloned as insert DNA for reporter vector construction using primers containing NotI sites (Supplementary Table 2). The insert DNA and psiCHECK-2 Dual luciferase reporter vector (C8021, Promega, Madison, MA) were digested by NotI (R0189, NEB, Ipswich, MA, USA) and then ligated. To mutate two miR-145 seed regions on the SEMA3A 3'-UTR, a Q5 Site-Directed Mutagenesis Kit (E0554S, NEB) was used. The primers for mutagenesis were designed by NEBaseChanger (http://nebasechanger.neb.com/, Supplementary Table 2). For amplification, the constructed vector was transformed into E. coli (JM109, L2005, Promega) and cultured overnight at $37^{\circ} \mathrm{C}$. The amplified vectors were harvested by the NucleoBond Xtra Midi kit (740410.10, Macherey-Nagel, Dueren, Germany) according to the manufacturer's protocol and then purified by Spin-X (CLS8160, Sigma-Aldrich, St Louis, MO, USA).

\section{Dual luciferase reporter assay}

HEK293T cells were seeded overnight at $5 \times 10^{4}$ cells per well in a 24 -well plate. The next day, the cells were transfected with $10 \mathrm{ng}$ of constructed vector plus $50 \mathrm{~nm}$ of negative control mimic (AllStars Negative Control siRNA, Qiagen, SI03650318) or $50 \mathrm{~nm}$ of hsa-miR-145 mimic (Qiagen, MSY0000437) by Lipofectamine 3000 according to the manufacturer's protocol. The cells were harvested 24 or $48 \mathrm{~h}$ after transfection. A Dual-Glo luciferase reporter assay kit (E2920, Promega) was used for luciferase analysis according to the manufacturer's instruction. Luciferase activity was measured with SpectraMax L (Molecular Devices, Sunnyvale, CA, USA).

\section{Statistical analysis}

Data in the in vitro functional experiments are expressed as the mean \pm s.d. Comparisons of the numerical data between groups were performed by the $t$-test or Mann-Whitney $U$-test. $P$-values $<0.05$ were considered statistically significant. 
a

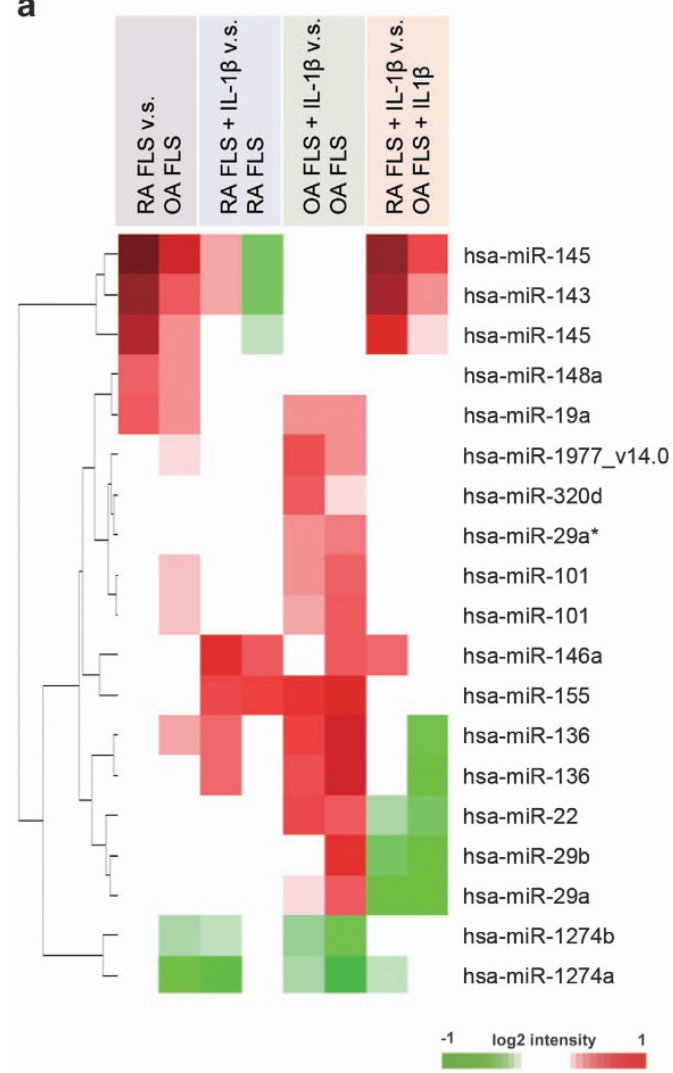

b
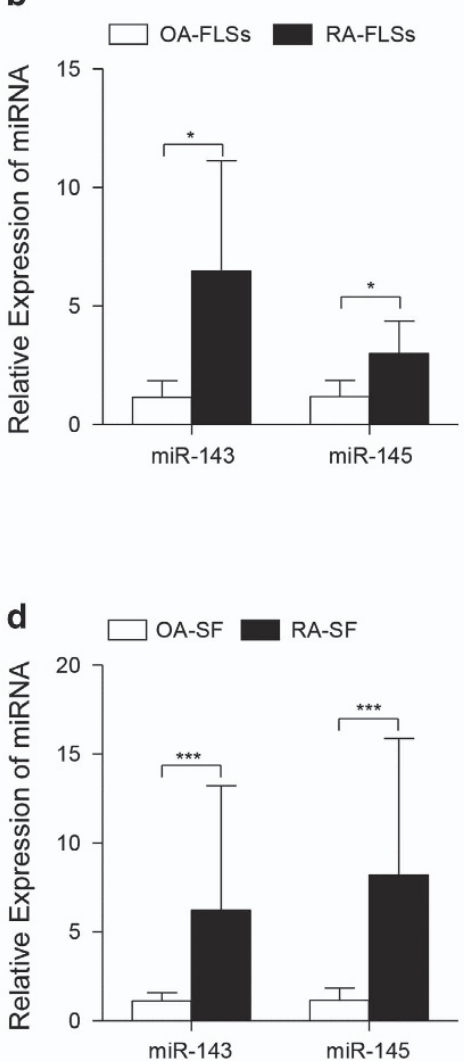

C

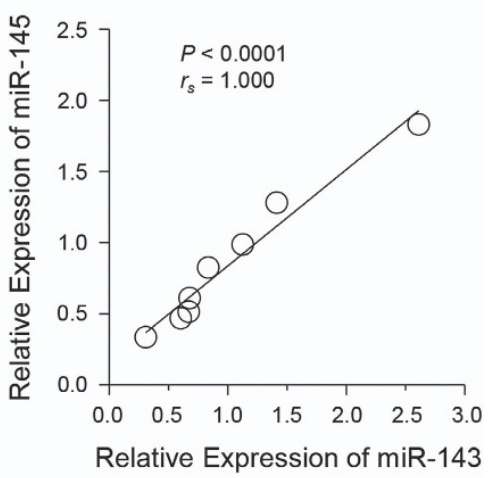

e

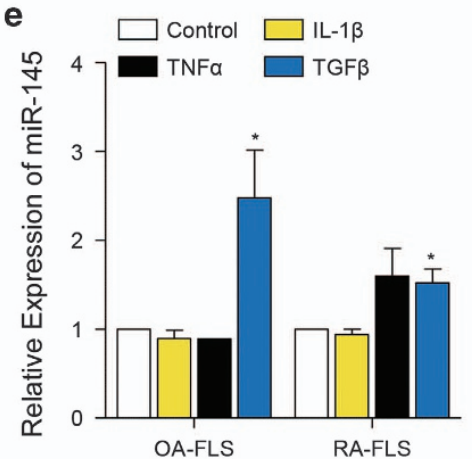

Figure 1 microRNA profiling in RA-FLSs. (a) Heatmap of microarray data showing DEmiRs among RA-FLSs versus OA-FLSs, RA-FLSs with IL-1 $\beta$ versus RA-FLSs, OA-FLSs with IL-1 $\beta$ versus OA-FLSs and RA-FLSs with IL-1 $\beta$ versus OA-FLSs with IL-1 $\beta$. Red and green denote high and low expression of DEmiRs, respectively; red and green denote high and low expression of DEGs, respectively. (b) Higher levels of miR-143 and miR-145 in RA-FLSs $(n=5)$ than in OA-FLSs $(n=5)$ as determined by quantitative real-time PCR; 5.6 -fold, $P=0.031$ for miR-143; 2.6-fold, $P=0.028$ for miR-145. RUN6B_2 small nuclear RNA (snRNA) was used as an internal control. (c) Spearman correlation of relative expression of miR-143 and miR-145 in RA-FLSs $(n=8)$. (d) miR-143 and miR-145 levels in synovial fluids obtained from RA patients (RA-SF, $n=4)$ and OA patients (OA-SF, $n=5$ ) as determined by real-time PCR. (e) Increase in miR-145 expression in FLSs by TGF $\beta$ stimulation. The OA-FLSs $(n=4)$ and RA-FLSs $(n=4)$ were treated with IL-1 $\beta\left(1 \mathrm{ng} \mathrm{ml}^{-1}\right)$, TNF $\alpha\left(10 \mathrm{ng} \mathrm{ml}^{-1}\right)$ and TGF $\beta$ $\left(10 \mathrm{ng} \mathrm{ml}^{-1}\right.$ ) for $48 \mathrm{~h}$, and miR-145 expression was then determined by real-time PCR. The data show the mean $\pm \mathrm{s} . \mathrm{d}$. ${ }^{*} P<0.05$ and $* * * P<0.001$.

\section{Study approval}

This study was performed with the approval of the institutional review board (CUMC09U034).

\section{RESULTS}

miR-143 and miR-145 are highly expressed in RA-FLSs

To determine the differentially expressed microRNAs (DEmiRs) in RA-FLSs, we initially performed a microarray analysis. From the four comparisons, including RA-FLSs versus OA-FLSs, RA-FLSs with IL- $1 \beta$ versus RA-FLSs, OA-FLSs with IL- $1 \beta$ versus OA-FLSs and RA-FLSs with IL- $1 \beta$ versus OA-FLSs with IL-1 $\beta, 16$ DEmiRs were identified with significant up- or downregulation in one of those comparisons (Figure 1a). Of note, miR-143 and miR-145, out of 16 total DEmiRs, exhibited significantly higher expression in RA-FLSs compared to OA-FLSs regardless of IL-1 $\beta$ treatment (Figure 1a). We confirmed the higher expression levels of miR-143 and miR-145 in independent RA-FLSs by real-time PCR
(Figure 1b). Notably, we found a clear positive correlation of the expression levels between miR-143 and miR-145 (Figure 1c). Given that genes encoding miR-143 and miR-145 are located in the same cluster in chromosome $5,{ }^{13}$ this result suggests that miR-143 and miR-145 could be co-regulated in RA-FLSs. As miRNAs can be present in human biological fluids, including plasma, semen and synovial fluids, ${ }^{14-16}$ we investigated the levels of miR-143 and miR-145 in synovial fluids from RA patients (RA-SF) and OA patients (OA-SF). Consistent with the results from FLSs, miR-143 and miR-145 levels were also significantly higher in RA-SF than in OA-SF (Figure 1d); their mean levels in RA-SF were 5.6- and 7.1-fold higher than those in OA-SF, respectively.

Since microarray data showed that miR-143 and miR-145 expression levels were not affected by IL- $1 \beta$ stimulation, we investigated whether other pro-inflammatory cytokines could induce miR-143 or miR-145 in FLSs (Figure 1e). As expected, 
a

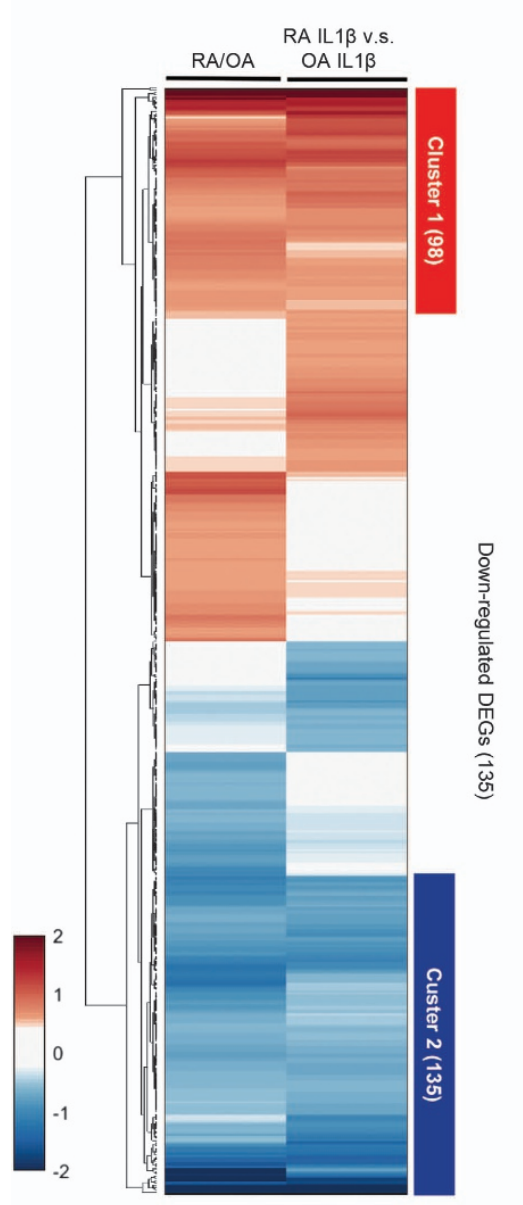

b
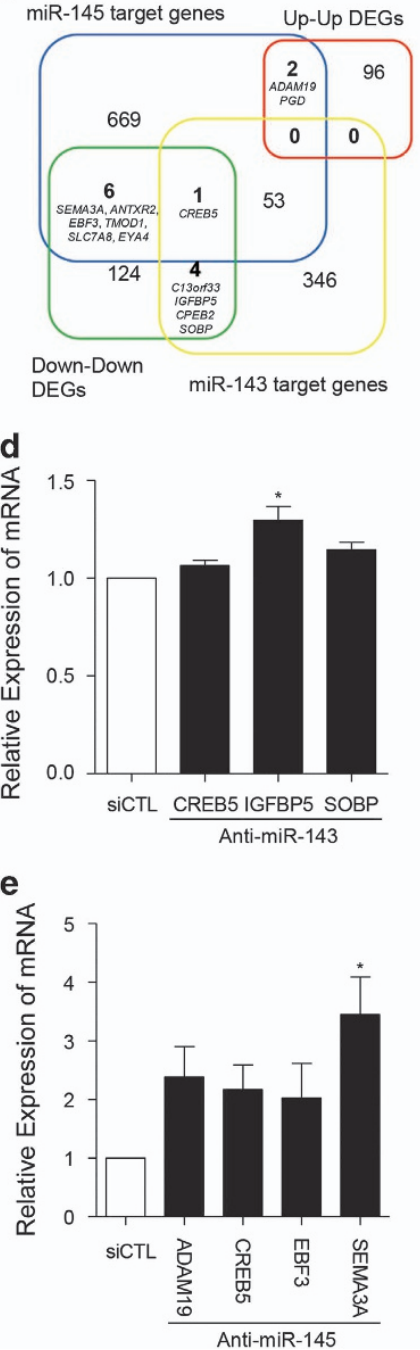

C

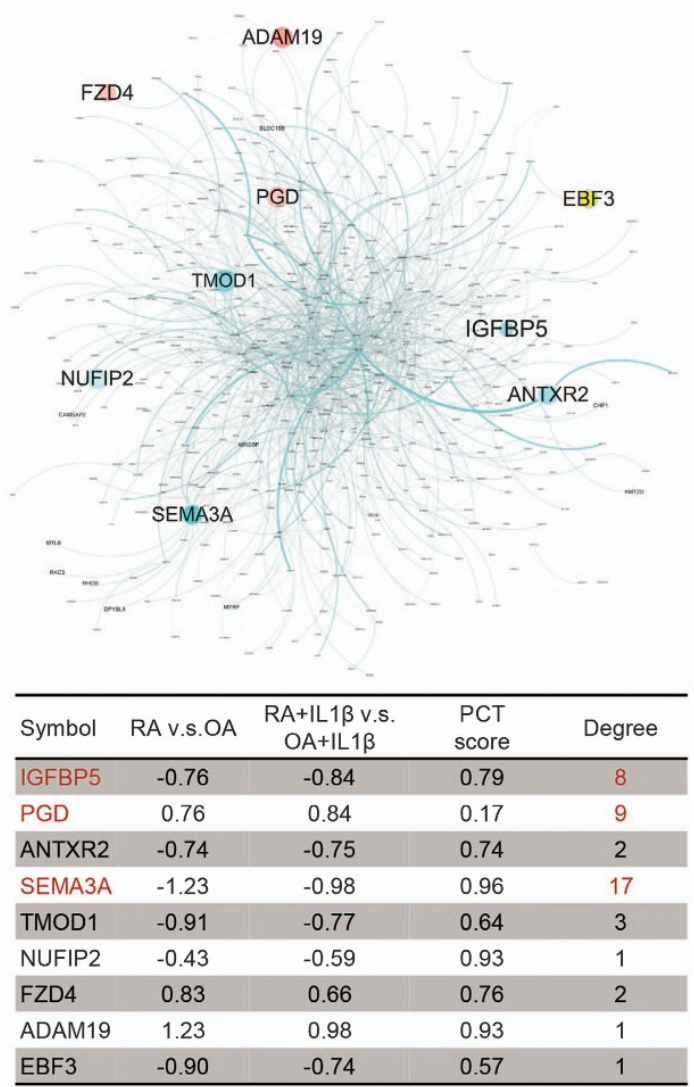

Figure 2 miR-143/145-target prediction and network model of the predicted targets in RA-FLSs. (a) Heatmap of 470 DEGs up- or downregulated in RA-FLSs irrespective of IL-1 $\beta$ stimulation. Compared profile sets were as follows: RA-FLSs versus OA-FLSs; RA-FLSs with IL-1 $\beta$ versus OA-FLSs with IL-1 $\beta$; RA-FLSs with IL-1 $\beta$ versus RA-FLSs; OA-FLSs with IL-1 $\beta$ versus OA-FLSs. Cluster 1 is a group of 98 DEGs that was upregulated in RA-FLSs and Cluster 2 represents 135 DEGs that were downregulated. The numbers in parenthesis refer to the number of included DEGs in each cluster. Red and blue denote high and low expression of DEGs, respectively (b) Venn diagram showing the relationship between two sets of predicted target genes for miR-143 and miR-145 and two DEG cluster sets of RA-FLSs versus OA-FLSs. The numbers of included genes are represented. (c) Network model of predicted target genes for miR-143 and miR-145 (miR-143: 404 genes, miR-145: 731 genes, shared: 54 genes, and total: 1081 genes). Node color refers to fold change of RA-FLSs versus OA-FLSs (red=up, cyan=down). Node size refers to $-\log 10 \mathrm{P}$ of RA-FLSs versus OA-FLSs. Edge thickness indicates edge betweenness (upper panel). The table in the lower panel shows the fold change, probability of conserved targeting (PCT) score, and the degree of interactions of potential target gene candidates in the network model. (d, e) Relative expression of six selected target DEGs in RA-FLSs transfected with miR-143 siRNA (d) or miR-145 siRNA (e), as determined by real-time PCR. GAPDH mRNA was used as an internal control. The data show the mean \pm s.d. ${ }^{*} P<0.05$.

there was no alteration in miR-145 levels with IL-1 $\beta$ treatment. TNF $\alpha$ stimulation also failed to upregulate miR-145 expression in FLSs. However, recombinant TGF $\beta$ significantly increased miR-145 expression in both OA-FLSs and RA-FLSs, which is consistent with a previous report that TGF $\beta$ upregulates miR-145 expression in vascular smooth muscle cells. ${ }^{17}$ Altogether, we demonstrated that miR-143 and miR-145 were highly expressed in RA-FLSs and that miR-145 expression in RA-FLSs was upregulated by TGF $\beta$ stimulation.

\section{Identification of target genes for miR-143 and miR-145 in RA-FLSs}

To identify potential target genes that could be regulated by miR-143 and miR-145 in RA-FLSs, we performed global transcriptomic profiling (GSE49604) using microarray analysis in RA-FLSs, including the FLSs from which the miRNA data were produced. ${ }^{10}$ We selected a set of DEGs through the comparisons of RA-FLSs versus OA-FLSs and RA-FLSs with IL- $1 \beta$ versus $O A-F L S$ s with IL- $1 \beta$. As a result, 470 genes were 

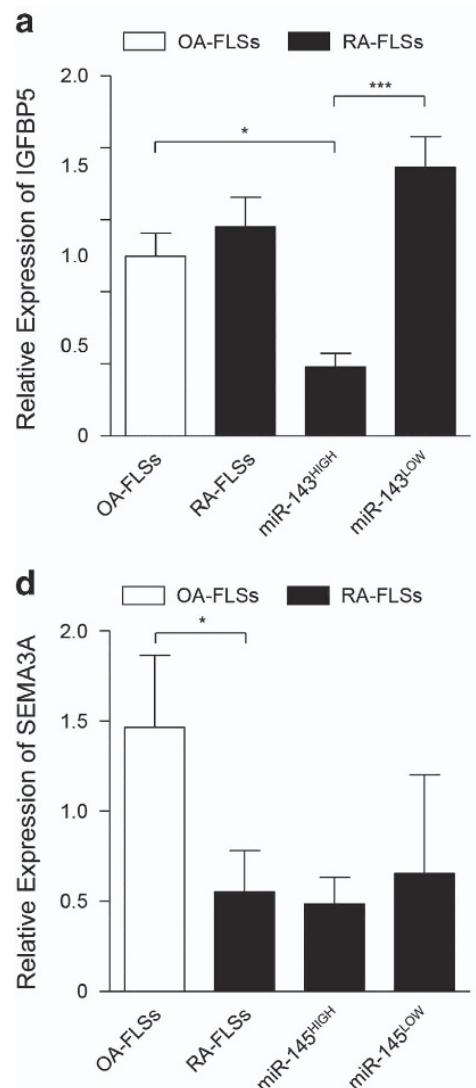

b

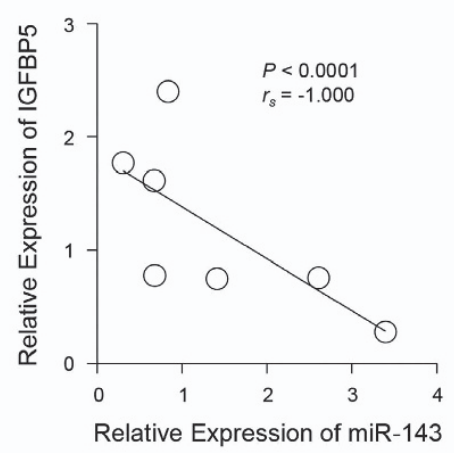

C

\begin{tabular}{lcc}
\hline Genes & $\begin{array}{c}\text { Kasperkovitz et al. } \\
\text { (ref. 19) }\end{array}$ & this study \\
\hline ACTA2 & -0.4 & -0.35 \\
COL3A1 & -0.2 & -0.25 \\
COL4A2 & -0.31 & -0.49 \\
POSTN & -0.25 & -0.71 \\
SPARC & -0.28 & -0.18 \\
CYR61 & -0.44 & -0.28 \\
SERPINH1 & -0.53 & -0.52 \\
CALD1 & -0.28 & -0.45 \\
\hline
\end{tabular}

e

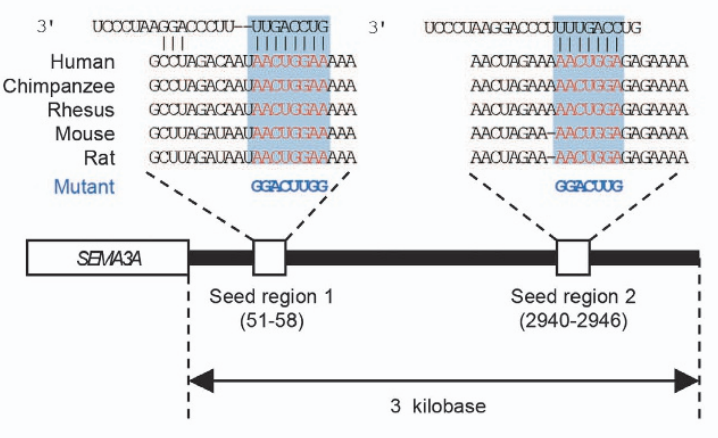

f

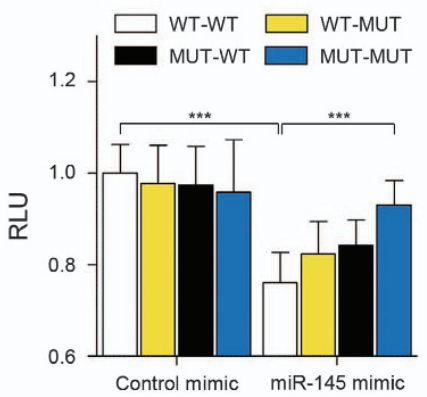

Figure 3 Validation of IGFBP5 and SEMA3A as miR-143 and miR-145 targets, respectively. (a) Comparison of IGFBP5 mRNA expression between RA-FLSs $(n=7)$ and OA-FLSs $(n=10)$, which was determined by real-time PCR. GAPDH mRNA was used as an internal control. miR-143 ${ }^{\mathrm{HIGH}}(n=3)$ and miR-143 ${ }^{\mathrm{LOW}}(n=4)$ indicate RA-FLS subsets with higher and lower expression of miR-143, respectively. (b) Negative correlation between miR-143 and IGFBP5 expression in RA-FLSs $(n=7)$. (c) Negative correlation of IGFBP5 gene expression levels with eight TFG $\beta$-responsive genes in RA-FLSs $(n=19)$ used in the previous study by Kasperkovitz et al. ${ }^{19}$ (GSE4061) and those $(n=6)$ in this study (GSE22956 and GSE49604). ACTA2, Actin, Alpha 2, Smooth Muscle, Aorta; COL3A1, Collagen Type III Alpha 1 Chain; COL4A2, Collagen Type IV Alpha 2 Chain; POSTN, Periostin; SPARC, secreted protein acidic and rich in cysteine; CYR61, Cysteine Rich Angiogenic Inducer 61; SERPINH1, Serine (or Cysteine) Proteinase Inhibitor, Clade H (Heat Shock Protein 47), Member 1 (Collagen Binding Protein 1); CALD1, Caldesmon 1. Values are Pearson's rho coefficients. (d) SEMA3A mRNA expression in RA-FLSs ( $n=7$ ) versus OA-FLSs $(n=10)$. miR-145 ${ }^{\mathrm{HIGH}}(n=3)$ and miR-145 ${ }^{\mathrm{LOW}}(n=4)$ indicate RA-FLS subsets with higher and lower expression of miR-145, respectively. (e) Sequence of miR-145 seed regions in the SEMA3A 3'-UTR. The mutation sequences are indicated in blue. (f) Relative normalized Renilla luciferase activity of reporter vector containing putative miR-145 binding sites (WT) or its mutated version (MUT). HEK293T cells were transfected with reporter vectors in the presence or absence of control mimic or synthetic miR-145 miRNA mimic. Single mutants of two putative miR-145-binding sites (seed regions) are indicated as WT-MUT or MUT-WT. A double mutant is denoted as MUT-MUT. RLU, relative light unit. The data show the mean \pm s.d. of three independent experiments. ${ }^{*} P<0.05 ;{ }^{* * *} P<0.001$.

selected that had significant differential expression in each of the comparisons. Hierarchical clustering of 470 DEGs displayed shared and distinct expression patterns (Figure 2a). Among those expression patterns, we focused on genes with a shared expression pattern (highlighted with red or blue bars to the right of heatmap), since both miR-143 and miR-145 were upregulated irrespective of IL-1 $\beta$ stimulation (Figure 2a). We then compared the two gene sets with shared up- or downregulation with predicted target gene lists of miR-143 and miR-145 that were obtained from TargetScan 6.0 (http:// www.targetscan.org/vert_60/). As a result, 12 genes (ADAM19, ANTXR2, C13orf33, CREB5, EBF3, EYA4, IGFBP5, NUFIP2, $P G D, S E M A 3 A, S O B P$ and TMOD1) were selected as potential candidates that could be up- or downregulated by miR-143 or miR-145 (Figure 2b).
To evaluate the target candidates, we utilized another approach to reconstruct a network model of 1081 genes that were predicted to be targets of miR-143 and miR-145 using TargetScan 6.0 (Figure 2c). We computed the degree of interaction of potential target gene candidates in the network model and identified three genes (IGFBP5, PGD and SEMA3A), which had more than five interactions with other genes in the network (Figure 2c, marked in red in the lower panel). The probability of conserved targeting (PCT) scores of the genes indicated that IGFBP5 and SEMA3A had higher PCT scores than PGD (Figure 2c, lower panel). Moreover, IGFBP5 and SEMA3A were significantly downregulated in RA-FLS with or without IL-1 $\beta$ stimulation, whereas $P G D$ was upregulated. Together, these results suggest that IGFBP5 and SEMA3A are the top two potential target candidates of miR-143 and 
miR-145 in RA-FLSs. To confirm the relationship between the miRNAs and potential targets, we tested whether specific antagomirs of miR-143 and miR-145 increase IGFBP5 and SEMA3A expression levels in RA-FLSs. Mean levels of SEMA3A and IGFBP5 mRNA were significantly increased by inhibition of miR-145 and miR-143, respectively (for SEMA3A, 3.45-fold, $P=0.02$; for IGFBP5, 1.31-fold, $P=0.02$ ) (Figure 2d and e), indicating that SEMA3A and IGFBP5 expression may be regulated by miR-143 and miR-145 in RA-FLSs, respectively.

\section{Validation of the miRNA-target relationship}

It has been demonstrated that miR-143 directly binds to the 3'-UTR of IGFBP5 mRNA and thereby inhibits IGFBP5 expression in a post-transcriptional manner. ${ }^{18}$ Here we found that, in contrast to OA-FLSs, RA-FLSs showed a high degree of heterogeneity in miR-143 expression and could be divided into two subgroups according to miR-143 expression: (1) RA-FLSs with high levels of miR-143 (miR-143 $\left.{ }^{\mathrm{HIGH}}\right)$ and (2) ones with a low level (miR-143 $\left.{ }^{\mathrm{LOW}}\right)$. To investigate the relationship between miR-143 and its target IGFBP5 in RA-FLSs, we first compared IGFBP5 expression in the two subgroups of
RA-FLSs. We found that IGFBP5 levels were significantly lower in the miR-143 ${ }^{\mathrm{HIGH}}$ RA-FLSs than in the miR-143 ${ }^{\mathrm{LOW}}$ RA-FLSs and OA-FLSs (Figure 3a). Moreover, consistent with a previous report, ${ }^{18}$ the expression of miR-143 and IGFBP5 mRNA in RA-FLSs displayed an inverse correlation (Figure 3b), suggesting that miR-143 negatively regulates IGFBP5 expression in RA-FLSs. An earlier study had described that RA-FLSs with low IGFBP5 expression displayed a myofibroblast phenotype with strong TGF $\beta$ signaling, ${ }^{19}$ which prompted us to investigate whether decreased IGFBP5 expression reflects a myofibroblast phenotype of RA-FLSs in our experimental setting. From microarray analysis, we found that IGFBP5 levels negatively correlated with expression levels of a variety of genes representing the myofibroblast phenotype with high TGF $\beta$ signaling, including ACTA2, COL3A1, COL4A2, POSTN, SPARC, CYR61, SERPINH1 and CALD1 (Figure 3c). Collectively, these results, together with a previous report $^{19}$ suggest that low IGFBP5 expression linked to miR-143 is correlated with the myofibroblast phenotype of RA-FLSs.

To date, there has been no report demonstrating an interaction between SEMA3A and miR-145. Presently, a

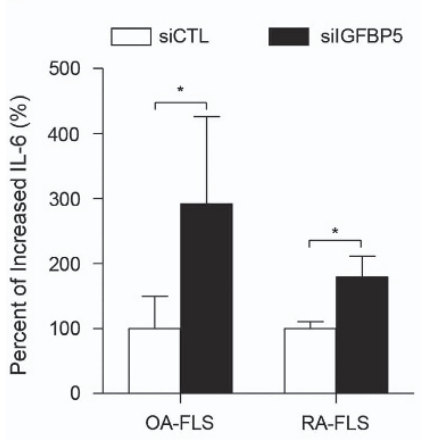

d

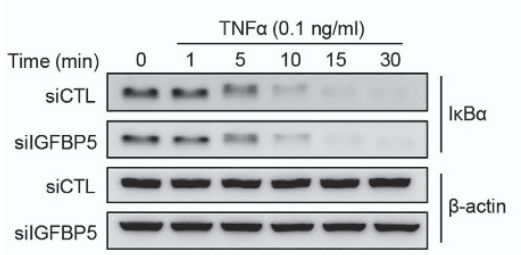

b

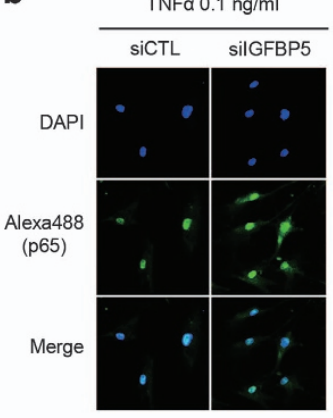

C

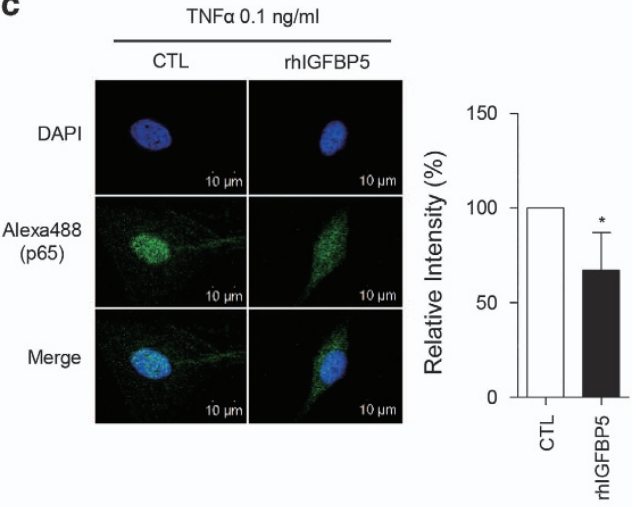


in contrast to IGFBP5 expression showing heterogeneity, a significantly lower expression of SEMA3A was consistently noted in RA-FLSs, independent of miR-145 expression (Figure 3d); this implied that other regulators might control SEMA3A levels in addition to miR-145. Therefore, it was unclear whether miR-145 directly bound to SEMA3A mRNA. To address this issue, we performed a dual-luciferase reporter assay using wild-type and mutant vector systems (Figure 3e). We found that a synthetic miR-145 mimic, but not a control mimic, significantly suppressed luciferase activity of the reporter vector expressing the wild-type 3 '-UTR (Figure 3f). By contrast, a single mutation in the putative two miR-145-binding sites made luciferase activity resistant to the miR-145 mimic (Figure 3f). Moreover, this resistance was further enhanced by co-transfection of a double mutant of the two predicted miR-145-binding sites with the miR-145 mimic, which strongly suggests that miR-145 directly binds to the 3'-UTR of SEMA3A mRNA (Figure 3f).
IGFBP5 suppresses TNF $\alpha$ signaling in RA-FLSs

Next, we sought to determine the effect of diminished IGFBP5 expression on RA-FLS function. IGFBP5 was originally identified as a regulator of insulin-like growth factor (IGF) signaling. ${ }^{20}$ A recent study demonstrated that IGFBP5 also inhibited TNF $\alpha$ signaling by binding to TNF $\alpha$ receptor 1 (TNFR1) in histiocytic lymphoma cells. ${ }^{21}$ Therefore, we investigated whether IGFBP5 suppresses $\mathrm{TNF} \alpha$ signaling in RA-FLSs. To this end, we first examined changes in TNF $\alpha$-stimulated IL- 6 production, a representative cytokine induced by $\mathrm{TNF} \alpha,{ }^{22}$ in FLSs following IGFBP5 siRNA (silGFBP5) transfection. We found that TNF $\alpha$-induced IL-6 production was significantly higher in siIGFBP5-transfected FLSs than in control siRNA-transfected FLSs (1.8-fold change, $P=0.029$ for RA-FLSs; 2.9 -fold change, $P=0.033$ for OA-FLSs) (Figure 4a).

It is well known that $\mathrm{TNF} \alpha$ activates the NF- $\mathrm{KB}$ signaling pathway through $\mathrm{I} \kappa \mathrm{B} \alpha$ degradation and p65 translocation. ${ }^{23}$ Thus, we tested the effect of IGFBP5 on p65 translocation and a
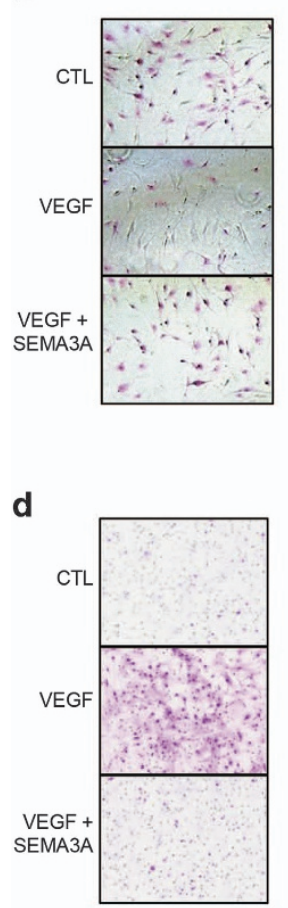
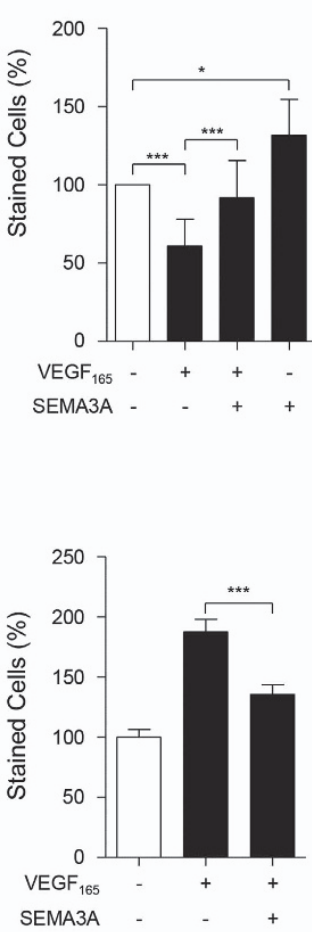

b

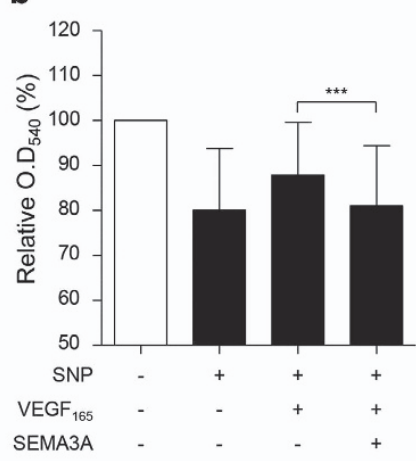

e

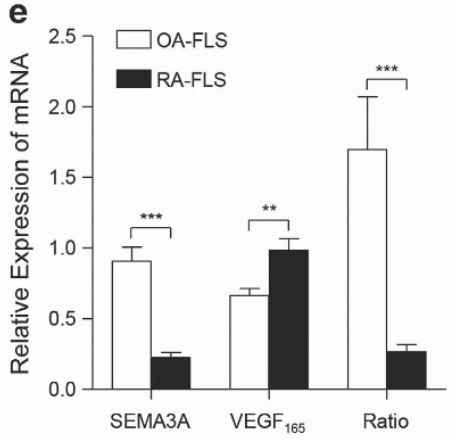

c
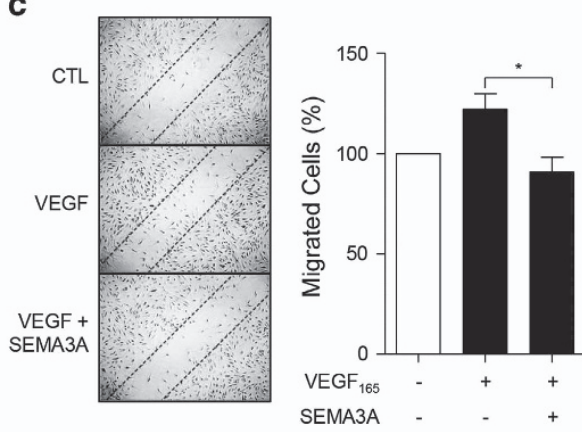

f

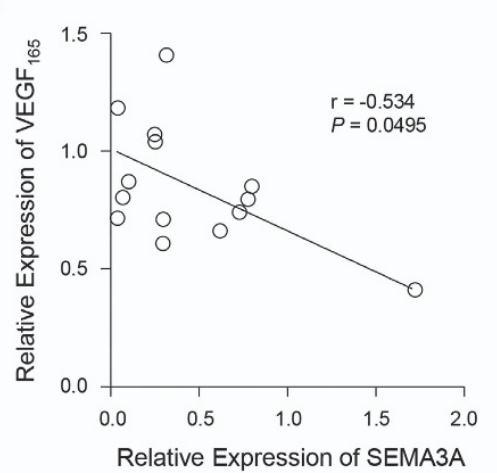

Figure 5 SEMA3A antagonizes anti-apoptotic and pro-migratory effects of VEGF 165 in RA-FLSs. (a, b) SEMA3A suppression of a VEGF $_{165}$-induced increase in FLS viability. (a) Microscopic analysis of APOPercentage-stained RA-FLSs. RA-FLSs ( $n=3$ ) were cultured with VEGF $165\left(200 \mathrm{ng} \mathrm{ml}^{-1}\right)$ in the presence or absence of SEMA3A (200 $\mathrm{ng} \mathrm{ml}-1$ ). After $12 \mathrm{~h}$, the number of stained (apoptotic) cells were counted and presented as a percentage. A representative of three independent experiments is shown in the left panel. (b) RA-FLSs $(n=3)$ were cultured in $0.1 \%$ FBS DMEM with VEGF $165\left(200 \mathrm{ng} \mathrm{ml}^{-1}\right)$ in the presence or absence of SEMA3A (200 $\left.\mathrm{ng} \mathrm{ml}^{-1}\right)$ and treated with sodium nitroprusside (SNP, $1 \mathrm{~mm}$ ) for $12 \mathrm{~h}$. Cell viability was assessed by the MTT (tetrazolium) assay. (c, d) SEMA3A inhibition of VEGF $_{165}$-induced FLS migration and chemotaxis. RA-FLSs were cultured with VEGF $165\left(200 \mathrm{ng} \mathrm{ml}^{-1}\right)$ in the presence or absence of SEMA3A (100 $\mathrm{n} \mathrm{ml}^{-1}$ ) for $12 \mathrm{~h}$. In the wound migration assay (c), cells migrating beyond the guide line were counted and photographed; a representative of three independent experiments is shown in the left panel. For the invasion assay (d), RA-FLSs $(n=4)$ were incubated in a Matrigel chamber and the number of migrated cells on the lower surface of the membrane was counted after staining with Diff-Quik. (e) Relative expression of SEMA3A and $\mathrm{VEGF}_{165}$ mRNA in RA-FLSs $(n=9)$ and OA-FLSs $(n=5)$, as determined by real-time PCR. A ratio of SEMA3A to VEGF 165 mRNA expression was calculated for each FLS. (f) Correlation between SEMA3A and VEGF 165 mRNA in OA-FLSs $(n=5)$ and RA-FLSs $(n=9)$. The bar graphs in a-e show the mean \pm s.d. ${ }^{*} P<0.05 ;{ }^{* *} P<0.01 ; * * * P<0.001$. 
IкB $\alpha$ degradation triggered by TNF $\alpha$. We found that the TNF $\alpha$-induced p65 translocation to the nucleus was significantly increased by transfection of siIGFBP5 into RA-FLSs (Figure 4b). Conversely, recombinant human IGFBP5 (rhIGFBP5) significantly decreased p65 translocation to the nucleus (Figure 4c). Moreover, as shown in Figure $4 \mathrm{~d}$ and $\mathrm{f}$, the IкB $\alpha$ degradation rate in RA-FLSs was increased by the knockdown of IGFBP5 transcripts. Conversely, in the presence of rhIGFBP5, the degradation rate of IкB $\alpha$ induced by TNF $\alpha$ was significantly decreased (Figure $4 \mathrm{e}$ and f). Together, these results suggest that IGFBP5 inhibits TNF $\alpha$-induced pro-inflammatory signals in RA-FLSs, suppressing NF- $\mathrm{kB}$ activity and IL-6 production. Therefore, the decreased IGFBP5 expression linked to high miR-143 noted in RA-FLSs may contribute to the increased downstream activation of TNF $\alpha$ signaling.

\section{SEMA3A inhibits the effect of VEGF165 in RA-FLSs}

SEMA3A acts as a chemo-repellent and a competitive inhibitor of $\mathrm{VEGF}_{165}$ through binding on its receptor, neuropilin-1. ${ }^{24}$ We previously demonstrated that the interaction of $\mathrm{VEGF}_{165}$ with neuropilin-1 protects RA-FLSs from apoptotic death by regulating $\mathrm{Bcl}-2$ expression and Bax translocation. ${ }^{25}$ Moreover, SEMA3A was shown to block the $\mathrm{VEGF}_{165}$-induced increase in the survival and migration of breast carcinoma cells. ${ }^{26} \mathrm{We}$ therefore investigated whether SEMA3A has an inhibitory role in the survival of RA-FLSs as a VEGF 165 antagonist. We found that recombinant human SEMA3A (rhSEMA3A) treatment inhibited the anti-apoptotic effects of VEGF 165 in RA-FLSs (Figure 5a). Moreover, in the absence of recombinant $\mathrm{VEGF}_{165}$, rhSEMA3A significantly increased the number of apoptotic RA-FLSs (Figure 5a). Given our previous finding that endogenous VEGF $_{165}$ secreted from RA-FLSs contributes to maintenance of cell survival, ${ }^{25}$ such an increase may be due to the inhibition of endogenous VEGF $_{165}$ by SEAM3A, although it may also be hypothesized that SEMA3A itself induces FLS

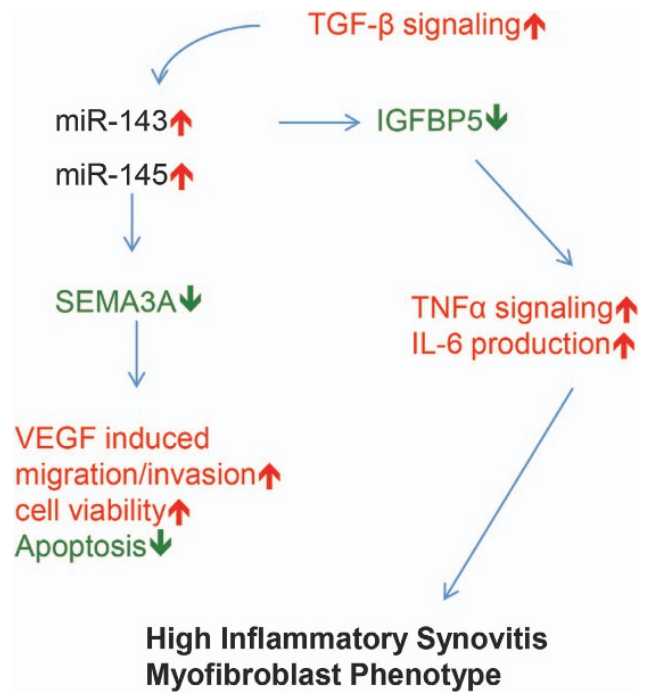

Figure 6 Hypothetical model for the role of miR-143 and miR-145 in RA-FLSs. apoptosis independent of VEGF 165 . Moreover, RA-FLS death induced by sodium nitroprusside (SNP), an apoptosis inducer, was prevented by the addition of recombinant $\mathrm{VEGF}_{165}$, which was nearly completely abrogated by co-treatment with SEMA3A, as determined by MTT assay (Figure 5b). Together, these observations indicate that SEMA3A promotes RA-FLS apoptosis by inhibiting $\mathrm{VEGF}_{165}$ action.

Previously, we also have shown that $\mathrm{VEGF}_{165}$ promotes RA-FLS adhesion and migration, which are essential properties of RA-FLSs for progression of RA. ${ }^{27}$ As seen in Figure $5 c$, SEMA3A inhibited the $\mathrm{VEGF}_{165}$-induced increase in wound migration of RA-FLSs. Moreover, the $\mathrm{VEGF}_{165}$-promoted invasion of RA-FLSs in a Matrigel-plugged chamber was also almost completely blocked by co-treatment with SEMA3A (Figure 5d). These observations demonstrated that, in addition to its pro-apoptotic activity, SEMA3A suppresses the $\mathrm{VEGF}_{165}$-induced increase in migration and invasion of RA-FLSs.

Intriguingly, measuring the simultaneous levels of $\mathrm{VEGF}_{165}$ and SEMA3A mRNA $n$ FLSs showed that the ratio of VEGF 165 to SEMA3A was significantly higher in RA-FLSs than in OA-FLSs (Figure 5e), which demonstrates the relative overexpression state of VEGF 165 compared to SEMA3A in RA-FLSs. Furthermore, SEMA3A mRNA levels negatively correlated with VEGF $_{165}$ mRNA levels in FLSs (Figure 5f), suggesting that RA-FLSs are intrinsically more susceptible to the anti-apoptotic and pro-migratory activities of VEGF $_{165}$.

Taken together, these data suggest that reduced SEMA3A linked to miR-145 overexpression in RA-FLSs leads to increased cell survival and increased migratory and invasive potential, resulting in tumor-like features for RA-FLSs.

\section{DISCUSSION}

Over the past decade, miRNAs have emerged as strong epigenetic regulators controlling pathological phenotypes associated with diseases involving cancer and autoimmune diseases. ${ }^{4}$ As miRNAs target multiple genes at the same time, it is difficult to identify the target genes responsible for miRNA-induced functional outputs. ${ }^{28,29}$ Thus, comprehensive profiling of both mRNA and miRNA expression levels is necessary to better predict target genes of miRNAs, providing greater insight into functional associations between mRNA and miRNA. ${ }^{30}$ In this study, we performed an integrated analysis of miRNA and mRNA profiles in RA-FLSs versus OA-FLSs, which facilitated the discovery of potential miRNA-target relationships. Moreover, network analysis of the predicted target genes provided the rationale for prioritizing the target genes. As a result, we found previously unidentified miRNAs, namely miR-143 and miR-145, that were upregulated in RA-FLSs compared to OA-FLSs. We also established a model for DEGs potentially regulated by miR-143 and miR-145 using TargetScan and successfully identified their targets, IGFBP5 and SEMA3A.

miR-143 and miR-145 are co-expressed miRNAs that are well-known potential tumor suppressors. ${ }^{31}$ In many cases, miR-143 and miR-145 are lower in expression levels in cancer 
cells than in normal cells, ${ }^{32,33}$ as they target numerous oncogenes. ${ }^{13,34}$ Interestingly, a recent study has demonstrated that miR-143 and miR-145 are highly expressed in mesenchymal cells, including fibroblasts and smooth muscle cells, rather than epithelial cells, ${ }^{35}$ which suggests a cell-type-specific expression of miR-143 and miR-145 in primary cells. In the present study, we validated the high expression of miR-143 and miR-145 in independent RA-FLSs. This implies that the functional roles of miR-143 and miR-145 in RA-FLS may be different from those of various cancer cells. Moreover, miR-145 expression in RA-FLSs was increased by TGF $\beta$ stimulation. Given the elevated TGF $\beta$ in RA joints, this observation supports our results demonstrating increased miR-145 expression in RA-FLSs.

miR-143 directly binds to the 3'-UTR of IGFBP5 mRNA, directing reprogramming of fibroblasts into smooth muscle cells. ${ }^{18}$ A previous study of the gene expression profiles of RA-FLSs showed that IGFBP5 levels in RA-FLSs negatively correlated with high inflammatory synovitis and a myofibroblast phenotype. ${ }^{19}$ However, it is still unclear how IGFBP5 expression is regulated in RA-FLSs and whether it directly contributes to RA pathology. In the present study, IGFBP5 expression was heterogeneous across the RA-FLS population, as it was significantly lower in miR-143 ${ }^{\mathrm{HIGH}} \mathrm{RA}-\mathrm{FLSs}$ than in miR-143 ${ }^{\text {LOW }}$ RA-FLSs and OA-FLSs. In addition, IGFBP5 levels negatively correlated with genes representing a myofibroblast phenotype. These results, together with a previous report, ${ }^{18}$ suggest that decreased IGFBP5 expression is epigenetically regulated as a miR-143 target and presumably is involved in the high inflammatory and myofibroblast phenotype of RA-FLSs. To address this issue, we tested the pro-inflammatory responses of RA-FLSs under IGFBP5deficient and -excess conditions. We demonstrated that IGFBP5 deficiency resulted in upregulation of TNF $\alpha$-induced NF- $\mathrm{KB}$ signaling in RA-FLSs, promoting IL-6 production. Conversely, treatment with recombinant IGFBP5 mitigated the TNF $\alpha$ downstream signaling. Collectively, these data suggest that IGFBP5-deficiency, linked to high miR-143 expression levels, renders RA-FLSs sensitive to TNF $\alpha$ (Figure 6). Given the high concentrations of TNF $\alpha$ in RA joints, an altered expression of IGFBP5 by high miR-143 levels in RA-FLSs may contribute to RA progression by promoting $\mathrm{TNF} \alpha$-induced NF- $\kappa \mathrm{B}$ activation and IL- 6 production, culminating in high inflammatory synovitis (Figure 6).

We have shown that VEGF $_{165}$ is highly expressed in RA-SF and RA-FLSs, increasing survival and migration of RA-FLSs by binding to neuropilin-1. ${ }^{25,27}$ By contrast, SEMA3A, a wellknown competitive inhibitor of $\mathrm{VEGF}_{165}$, is rarely expressed in RA synovial tissues, ${ }^{36}$ which raises the intriguing hypothesis that an imbalance between VEGF $_{165}$ and SEMA3A expression causes synovial hyperplasia and angiogenesis, that is, the pannus formation, through a lifting of the block on $\mathrm{VEGF}_{165}$. SEMA3A was originally identified as an axonal guidance molecule, ${ }^{37}$ and it also suppresses cell migration and apoptosis by sharing receptors with VEGF $_{165}$ in neural progenitor cells. ${ }^{38}$ A study has demonstrated that decreased SEMA3A expression in RA synovia negatively correlates with disease activity score 28 (DAS28) and high inflammatory synovitis. ${ }^{36}$ However, it remains unclear how SEMA3A expression is regulated in RA synovia and whether it can antagonize the anti-apoptotic and pro-migratory activity of $\mathrm{VEGF}_{165}$ in RA-FLSs. Here we identified for the first time that the $3^{\prime}$-UTR of SEMA3A mRNA is a direct target of miR-145 using a luciferase reporter assay. Moreover, we confirmed an imbalance between VEGF $_{165}$ and SEMA3A expression in RA-FLSs and found that SEMA3A strongly suppressed the $\mathrm{VEGF}_{165}$-induced increase in survival, migration and invasion of RA-FLSs. Given the high concentrations of $\mathrm{VEGF}_{165}$ in RA joints, ${ }^{39}$ these results suggest that decreased SEMA3A linked to high miR-145 increases RA-FLS sensitivity to $\mathrm{VEGF}_{165}$ stimulation (Figure 6), adding another layer of $\mathrm{VEGF}_{165}$-mediated FLS pathology.

The pathologic hallmark of RA is the formation of an invasive pannus, which consists of synovial hyperplasia and extensive angiogenesis. RA-FLSs epigenetically acquire an aggressive phenotype and become the spearhead of the inflamed synovium, mediating chronic inflammation and destruction of the joints. ${ }^{40}$ Such aggressive behavior of RA-FLSs is regulated by diverse molecular mechanisms, including miRNAs. Here we provide new insights into the relationships between miR-143 and miR-145 signatures and their functional targets, IGFBP5 and SEMA3A, and define the pathological phenotypes of RA-FLSs, including pro-inflammatory, anti-apoptotic, pro-migratory and proinvasive features. This study also offers new possibilities with regard to miR-143- and miR-145-based approaches for targeting RA-FLSs and for treating RA.

\section{CONFLICT OF INTEREST}

The authors declare no conflict of interest.

\section{ACKNOWLEDGEMENTS}

We thank the members of the Center for Integrative Rheumatoid Transcriptomics and Dynamics at the Catholic University of Korea for their assistance. This work was supported by a grant from the National Research Foundation of Korea (NRF) funded by the Ministry of Education, Science and Technology (2015R1A3A2032927).

1 Firestein GS. Evolving concepts of rheumatoid arthritis. Nature 2003; 423: 356-361.

2 Firestein GS. Invasive fibroblast-like synoviocytes in rheumatoid arthritis. Passive responders or transformed aggressors? Arthritis Rheum 1996; 39: 1781-1790.

3 Bartok B, Firestein GS. Fibroblast-like synoviocytes: key effector cells in rheumatoid arthritis. Immunol Rev 2010; 233: 233-255.

4 Furer V, Greenberg JD, Attur M, Abramson SB, Pillinger MH. The role of microRNA in rheumatoid arthritis and other autoimmune diseases. Clin Immunol 2010; 136: 1-15.

5 Gao J, Zhou XL, Kong RN, Ji LM, He LL, Zhao DB. microRNA-126 targeting PIK3R2 promotes rheumatoid arthritis synovial fibro-blasts proliferation and resistance to apoptosis by regulating PI3K/AKT pathway. Exp Mol Pathol 2016; 100: 192-198.

6 Li XF, Shen WW, Sun YY, Li WX, Sun ZH, Liu YH et al. MicroRNA-20a negatively regulates expression of NLRP3-inflammasome by targeting TXNIP in adjuvant-induced arthritis fibroblast-like synoviocytes. Joint Bone Spine 2016; 83: 695-700. 
7 Philippe L, Alsaleh G, Pichot A, Ostermann E, Zuber G, Frisch B et al. MiR-20a regulates ASK1 expression and TLR4-dependent cytokine release in rheumatoid fibroblast-like synoviocytes. Ann Rheum Dis 2013; 72: 1071-1079.

8 Yang S, Yang Y. Downregulation of microRNA221 decreases migration and invasion in fibroblastlike synoviocytes in rheumatoid arthritis. Mol Med Rep 2015; 12: 2395-2401.

9 Bolstad BM, Irizarry RA, Astrand M, Speed TP. A comparison of normalization methods for high density oligonucleotide array data based on variance and bias. Bioinformatics 2003; 19: 185-193.

10 You S, Yoo SA, Choi S, Kim JY, Park SJ, Ji JD et al. Identification of key regulators for the migration and invasion of rheumatoid synoviocytes through a systems approach. Proc Natl Acad Sci USA 2014; 111: 550-555.

11 Hwang D, Rust AG, Ramsey S, Smith JJ, Leslie DM, Weston AD et al. A data integration methodology for systems biology. Proc Natl Acad Sci USA 2005; 102: 17296-17301.

12 Huang da W, Sherman BT, Lempicki RA. Systematic and integrative analysis of large gene lists using DAVID bioinformatics resources. Nat Protoc 2009; 4: 44-57.

13 Elia L, Quintavalle M, Zhang J, Contu R, Cossu L, Latronico MV et al. The knockout of miR-143 and -145 alters smooth muscle cell maintenance and vascular homeostasis in mice: correlates with human disease. Cell Death Differ 2009; 16: 1590-1598.

14 Mitchell PS, Parkin RK, Kroh EM, Fritz BR, Wyman SK, Pogosova-Agadjanyan EL et al. Circulating microRNAs as stable bloodbased markers for cancer detection. Proc Natl Acad Sci USA 2008; 105: 10513-10518.

15 Murata K, Yoshitomi H, Tanida S, Ishikawa M, Nishitani K, Ito $\mathrm{H}$ et al. Plasma and synovial fluid micrornas as potential biomarkers of rheumatoid arthritis and osteoarthritis. Arthritis Res Ther 2010; 12: R86.

16 Hanson EK, Lubenow $\mathrm{H}$, Ballantyne J. Identification of forensically relevant body fluids using a panel of differentially expressed microRNAs. Anal Biochem 2009; 387: 303-314.

17 Climent M, Quintavalle M, Miragoli M, Chen J, Condorelli G, Elia L. TGFbeta triggers miR-143/145 transfer from smooth muscle cells to endothelial cells, thereby modulating vessel stabilization. Circ Res 2015; 116: 1753-1764.

18 Chivukula RR, Shi G, Acharya A, Mills EW, Zeitels LR, Anandam JL et al. An essential mesenchymal function for miR-143/145 in intestinal epithelial regeneration. Cell 2014; 157: 1104-1116.

19 Kasperkovitz PV, Timmer TC, Smeets TJ, Verbeet NL, Tak PP, van Baarsen LG et al. Fibroblast-like synoviocytes derived from patients with rheumatoid arthritis show the imprint of synovial tissue heterogeneity: evidence of a link between an increased myofibroblast-like phenotype and high-inflammation synovitis. Arthritis Rheum 2005; 52: 430-441.

20 Baxter RC, Martin JL. Binding proteins for the insulin-like growth factors: structure, regulation and function. Prog Growth Factor Res 1989; 1: 49-68.

21 Hwang JR, Huh JH, Lee Y, Lee SI, Rho SB, Lee JH. Insulin-like growth factor-binding protein-5 (IGFBP-5) inhibits TNF-alpha-induced NF-kappaB activity by binding to TNFR1. Biochem Biophys Res Commun 2011; 405: 545-551.

22 Li X, Makarov SS. An essential role of NF-kappaB in the 'tumor-like' phenotype of arthritic synoviocytes. Proc Natl Acad Sci USA 2006; 103: 17432-17437.

23 Beg AA, Finco TS, Nantermet PV, Baldwin AS Jr. Tumor necrosis factor and interleukin-1 lead to phosphorylation and loss of I kappa B alpha: a mechanism for NF-kappa B activation. Mol Cell Biol 1993; 13: 3301-3310.

24 Miao HQ, Soker S, Feiner L, Alonso JL, Raper JA, Klagsbrun M. Neuropilin-1 mediates collapsin-1/semaphorin III inhibition of endothelial cell motility: functional competition of collapsin-1 and vascular endothelial growth factor-165. J Cell Biol 1999; 146: 233-242.
25 Kim WU, Kang SS, Yoo SA, Hong KH, Bae DG, Lee MS et al. Interaction of vascular endothelial growth factor 165 with neuropilin-1 protects rheumatoid synoviocytes from apoptotic death by regulating $\mathrm{Bcl}-2$ expression and Bax translocation. J Immunol 2006; 177: 5727-5735.

26 Bachelder RE, Lipscomb EA, Lin X, Wendt MA, Chadborn NH, Eickholt BJ et al. Competing autocrine pathways involving alternative neuropilin-1 ligands regulate chemotaxis of carcinoma cells. Cancer Res 2003; 63: 5230-5233.

27 Kong JS, Yoo SA, Kim JW, Yang SP, Chae CB, Tarallo V et al. Antineuropilin-1 peptide inhibition of synoviocyte survival, angiogenesis, and experimental arthritis. Arthritis Rheum 2010; 62: 179-190.

28 Kuhn DE, Martin MM, Feldman DS, Terry AV Jr, Nuovo GJ, Elton TS Experimental validation of miRNA targets. Methods 2008; 44: 47-54.

29 Thomson DW, Bracken CP, Goodall GJ. Experimental strategies for microRNA target identification. Nucleic Acids Res 2011; 39: 6845-6853.

$30 \mathrm{Kim}$ KJ, Lee S, Kim WU. Applications of systems approaches in the study of rheumatic diseases. Korean J Intern Med 2015; 30: 148-160.

31 lio A, Nakagawa Y, Hirata I, Naoe T, Akao Y. Identification of non-coding RNAs embracing microRNA-143/145 cluster. Mol Cancer 2010; 9: 136.

32 Slaby O, Svoboda M, Fabian P, Smerdova T, Knoflickova D, Bednarikova M et al. Altered expression of miR-21, miR-31, miR-143 and miR-145 is related to clinicopathologic features of colorectal cancer. Oncology 2007; 72: 397-402.

33 Arndt GM, Dossey L, Cullen LM, Lai A, Druker R, Eisbacher M et al. Characterization of global microRNA expression reveals oncogenic potential of miR-145 in metastatic colorectal cancer. BMC Cancer 2009; 9: 374.

34 Pagliuca A, Valvo C, Fabrizi E, di Martino S, Biffoni M, Runci D et al. Analysis of the combined action of miR-143 and miR-145 on oncogenic pathways in colorectal cancer cells reveals a coordinate program of gene repression. Oncogene 2013; 32: 4806-4813.

35 Cordes KR, Sheehy NT, White MP, Berry EC, Morton SU, Muth AN et al. miR-145 and miR-143 regulate smooth muscle cell fate and plasticity. Nature 2009; 460: 705-710.

36 Takagawa S, Nakamura F, Kumagai K, Nagashima Y, Goshima Y, Saito T. Decreased semaphorin3A expression correlates with disease activity and histological features of rheumatoid arthritis. BMC Musculoskelet Disord 2013; 14: 40.

37 Kolodkin AL, Matthes DJ, Goodman CS. The semaphorin genes encode a family of transmembrane and secreted growth cone guidance molecules. Cell 1993; 75: 1389-1399.

38 Bagnard D, Vaillant C, Khuth ST, Dufay N, Lohrum M, Puschel AW et al. Semaphorin 3A-vascular endothelial growth factor-165 balance mediates migration and apoptosis of neural progenitor cells by the recruitment of shared receptor. J Neurosci 2001; 21: 3332-3341.

39 Lee SS, Joo YS, Kim WU, Min DJ, Min JK, Park SH et al. Vascular endothelial growth factor levels in the serum and synovial fluid of patients with rheumatoid arthritis. Clin Exp Rheumatol 2001; 19: 321-324.

40 Bottini N, Firestein GS. Duality of fibroblast-like synoviocytes in RA: passive responders and imprinted aggressors. Nat Rev Rheumatol 2013; 9: 24-33.

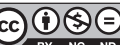

This work is licensed under a Creative Commons Attribution-NonCommercial-NoDerivs 4.0

International License. The images or other third party material in this article are included in the article's Creative Commons license, unless indicated otherwise in the credit line; if the material is not included under the Creative Commons license, users will need to obtain permission from the license holder to reproduce the material. To view a copy of this license, visit http://creativecommons.org/licenses/by-nc-nd/4.0/

Supplementary Information accompanies the paper on Experimental \& Molecular Medicine website (http://www.nature.com/emm) 